\title{
Ouabain, a Na-K-ATPase inhibitor, enhances wakefulness in rats
}

\author{
Zoltán Lelkes
}

Department of Physiology, Faculty of Medicine, University of Szeged, Dómtér 10, 6720 Szeged, Hungary

\section{H I G H L I G H T S}

- Sleep disturbances are common findings in bipolar affective disorder.

- Na-K-ATPase is implicated in sleep regulation.

- Blocking of Na-K-ATPase by ouabain is an animal model of bipolar affective disorder.

- Intracerebroventriculary administered ouabain enhanced wakefulness.

- Homeostatic feed back mechanisms may be impaired in bipolar affective disorder.

\section{A R T I C L E I N F O}

\section{Keywords:}

Sleep

Na-K-ATPase

Ouabain

Bipolar affective disorder

\begin{abstract}
A B S T R A C T
Sleep disturbances are common findings in bipolar affective disorder (BD), and they may be related to the pathophysiology of the disease. REM sleep deprivation, which has an antidepressant effect, increases Na-KATPase (NKA) activity. NKA might be involved in the induction of rebound sleep. Decrements in NKA are thought to be implicated in the pathophysiology of BD. A single intracerebroventricular (ICV) microinjection of ouabain (OUA), an NKA inhibitor, results in long-lasting suppression of NKA activity. It has been proposed to be used as a model of BD. To elucidate the role of NKA, we studied the effects of ICV administered OUA on the sleep-wake activity of rats. Rats were ICV injected with saline on the baseline day and $2.5 \mu \mathrm{L} 20 \mu \mathrm{M}(\mathrm{n}=8)$ or $2 \mathrm{mM}(\mathrm{n}=6)$ OUA on the subsequent OUA day. Saline was administered 1,4 , and 8 days after OUA injection. Sleep-wake activity was recorded for $12 \mathrm{~h}$ or $24 \mathrm{~h}$ post-injection. OUA enhanced wakefulness, but there was only little similarity between the changes in sleep and in BD after OUA treatment. These findings give only limited support for the NKA hypothesis of BD. Not the decrease in NKA activity itself that may be the important factor in the pathophysiology of $\mathrm{BD}$, but rather the dysfunction of those regulatory processes that influence NKA.
\end{abstract}

\section{Introduction}

Patients suffering from mood disorders often complain of sleep disturbances. Sleep abnormalities are similar in major depression and in bipolar affective disorder (BD) (Riemann et al., 2002): sleep latency is prolonged and sleep is fragmented. That is, there are many nocturnal awakenings, and sleep terminates early in the morning. During nonREM sleep, delta activity is decreased in the electroencephalogram (EEG), and the first non-REM sleep period is shortened, leading to a disinhibition and advanced occurrence of REM sleep, all representing changes in the sleep architecture (Reynolds and Kupfer, 1987). Hypersomnia has also been reported in the depressive phase of BD (Riemann et al., 2002). Furthermore, in mania, the amount of sleep is reduced to a great extent (Soreca, 2014). Sleep can additionally influence mood. Manipulations of sleep-wake activity (total or partial sleep deprivation, selective REM sleep deprivation, and phase advancement of the sleep-wake cycle) are effective in relieving depression (Reynolds and Kupfer, 1987; Riemann et al., 2002; Wirz-Justice and Van den Hoofdakker, 1999). Therefore, these findings may indicate a relationship between the underlying mechanisms of mood disorders and sleep disturbances.

Sodium and potassium-activated adenosine triphosphatase (NKA) dysfunction is thought to be implicated in the pathophysiology of BD, and decreases in NKA activity may be related to the mechanisms underlying both mania and bipolar depression (El-Mallakh, 1983; El Mallakh and Wyatt, 1995). Decreases in NKA activity have been reported in both mania and depression (Looney and El-Mallakh, 1997). NKA activity is also suppressed in an animal model of depression (Gamaro et al., 2003), and the levels of NKA suppressing endogenous cardiac steroids are increased in an animal model of mania (Hodes et al., 2016). In case of digitalis therapy, which suppresses NKA activity, both mania and depression may occur as a side effect (Keller and

E-mail address: lelkes.zoltan@med.u-szeged.hu. 
Frishman, 2003). The suppression of NKA activity by intracerebroventricular (ICV) administration of ouabain (OUA) has been proposed to be used as an animal model of BD. The effect of ICV administered OUA on motor activity depends on the way of recording. In an automated activity monitor, ICV injection of $5 \mu \mathrm{L} 1 \mathrm{mM}$ OUA results in a transient suppression of locomotor activity (Li et al., 1997). However, when motor activity is measured by recording the open field activity, the same treatment increases motor activity. When the open field test is repeated 9 days after the OUA administration, the motor activity is still high, indicating a long term action of OUA (Ruktanonchai et al., 1998). Both OUA induced suppression and enhancement of motor activity can be prevented by lithium treatment (ElMallakh et al., 2003; Li et al., 1997).

REM sleep deprivation enhances neuronal NKA via the release of noradrenaline and the activation of alpha-adrenergic receptors (Gulyani and Mallick, 1995). Noradrenaline and NKA may also be implicated in the changes of brain excitability following REM sleep deprivation (Mallick and Singh, 2011). Since excitation of alpha1-adrenergic receptors during REM sleep deprivation may contribute to the subsequent REM sleep rebound (Lelkes et al., 1994), it is possible that the activation of NKA may also be involved in the generation of rebound sleep. Basal forebrain cholinergic neurons and the release of adenosine are involved in the induction of rebound sleep (PorkkaHeiskanen et al., 1997). Such an adenosine release may be linked to the activation of NKA (Sims and Dale, 2014), which also supports the idea that activation of NKA might be involved in the mechanism of rebound sleep.

Thus, sleep disturbances are common findings in $\mathrm{BD}$, and they may be related to the pathophysiology of the disease. Besides, NKA might be involved in both the regulation of sleep-wake activity and the pathophysiology of BD. In order to clarify the role of NKA, the author studied the effects of suppression of NKA activity by ICV administered OUA on the sleep-wake activity of rats. In the present study, in addition to a marked awakening effect, epileptiform waves appeared in the EEG in response to a dose of $2.5 \mu \mathrm{L} 2 \mathrm{mM}$ OUA, equivalent to $5 \mu \mathrm{L} 1 \mathrm{mM}$ proposed as an animal model of BD. Since a lower dose, without any epileptogenic action, was thought to be a better model of BD, the effects of such a low dose were also tested. The dose had to be reduced substantially in order not to induce any epileptiform wave in the EEG. After testing several doses in pilot studies, $2.5 \mu \mathrm{L} 20 \mu \mathrm{M}$ OUA was proved not to induce any epileptogenic action. In the present study, this low dose only moderately enhanced wakefulness.

\section{Methods}

\subsection{Animals}

The experiments were carried out in male Sprague-Dawley rats weighing 300-400 $\mathrm{g}$ at the time of the sleep experiments. The animals were kept under conditions of controlled temperature $\left(24+1{ }^{\circ} \mathrm{C}\right)$ and lighting (lights on 8:30-20:30 h). Food and water were continuously available. All efforts were made to minimize the number of animals used and their suffering. Institutional guidelines for the care and use of research animals were followed and approved by the respective institutional committees. The procedures used in this study are in accordance with the EU directive for animal experiments.

\subsection{Surgery}

The surgeries were performed under ketamine-xylazine anesthesia ( 87 and $13 \mathrm{mg} / \mathrm{kg}$, respectively). The animals were implanted with stainless steel screw electrodes for EEG recordings over the cortex and the cerebellum (EEG screw 1: $1 \mathrm{~mm}$ from the midline, on the sutura coronarius; EEG screw 2: in the midline, $1.5-2 \mathrm{~mm}$ behind the lambda; and ground screw: over the parietal cortex). An ICV guide cannula was implanted into the left lateral cerebral ventricle stereotaxically
( $0.80 \mathrm{~mm}$ posterior from bregma, $1.4 \mathrm{~mm}$ lateral from midline, and $4.0 \mathrm{~mm}$ ventral from the surface of the skull (Paxinos and Watson, 1998)). The guide cannula was fixed with dental acrylic (Duracryl) to the skull. The location of the cannula was determined by the gravity method (sudden drop in pressure) during implantation. The drinking response to ICV injection of angiotensin was tested 3-4 days after surgery. The rats responding to angiotensin were used in further studies. After the experiments, trypan blue was injected ICV, and the ventricular system was checked for staining. The data refer to only the rats $(n=14)$ in which the dye stained both the third and the fourth ventricles and the cerebral aqueduct.

\subsection{Recording}

After the implantation operation, the rats lived in individual Plexiglas cages in the experimental chamber. They were allowed 7-10 days to recover. The animals were connected to recording cables in order to habituate them to the recording conditions. The recording cables were attached to commutators. The motor activity was assessed by means of recording potentials generated in electromagnetic transducers activated by movements of the cables (as performed previously, cf., Lelkes et al., 1987). Cables from the commutators and electromagnetic transducers were connected to amplifiers. The digitized (128Hz sampling rate) signals of the EEG and motor activity were collected by computers in an adjacent room. For scoring, the EEG and motor activity signals were restored on the computer screen. EEG power spectra were calculated by fast Fourier transformation (as performed previously in this laboratory, cf. Obál et al., 1999). The states of vigilance were determined for 8-s epochs by the usual criteria (used previously: Lelkes et al., 1987) as non-REM sleep (high-amplitude EEG slow waves, lack of body movement, predominant EEG power in the delta range); REM sleep (highly regular EEG theta activity with corresponding high theta power, and general lack of body movements with occasional twitches); and wakefulness (low-amplitude EEG, less regular theta activity than during REM sleep, and frequent body movements).

\subsection{Experimental protocol}

After the surgery, the rats were allowed to recover and habituate to the experimental conditions. Then, they received an ICV microinjection of $2.5 \mu \mathrm{L} \mathrm{SAL}$ on the baseline day, the same volume of $20 \mu \mathrm{M}(n=8)$ or $2 \mathrm{mM}(n=6)$ OUA on the subsequent OUA day and SAL on the following post-OUA days 1,4 and 8 . The microinjections were given into the lateral ventricle in dim light (lasting for 5-10 min) 5-15 min before light onset. The sleep-wake activity was recorded for 12 (i.e., during the light phase of each recording day; in case of the $2 \mathrm{mM}$ OUA treated rats) or $24 \mathrm{~h}$ (in case of the $20 \mu \mathrm{M}$ OUA treated rats) post-injection.

\subsection{Statistics}

The percentage of time spent in each vigilance state and the slow wave activity (SWA, EEG delta power, 0.5-4 Hz) during non-REM sleep were calculated for consecutive 3-h time blocks. These 3 -h values among the various (baseline, OUA post-OUA) recording days were compared by means of two-way analysis of variance (ANOVA) for repeated measures. The recording day effect (treatment effect, i.e. differences among the baseline, OUA and post-OUA days) and the time of day effect (variations in 3-h values across the day) were the two factors included in the ANOVA. In general, the F statistics are reported only for the recording day (treatment) effect and for the interactions between the recording day and the time of day factors when the differences were proved to be statistically significant. The Bonferroni test was used for post hoc comparisons. The numbers of wake, non-REM and REM sleep epochs for the 12-h recording periods and REM sleep latencies were calculated. Since the sleep-wake activity of animals is polyphasic, and not each non-REM sleep episode is followed by REM sleep, it is difficult 
to measure REM sleep latency in rats. REM sleep latency was defined as the delay between the onset of the 1st at least 120-s long epoch of sleep and the 1st at least 40-s long REM sleep epoch of the light phase. (In an earlier study, when sleep-wake activity was scored in 40-sec epochs, we defined REM sleep latency as the time between the onset of light and the occurrence of the 1st episode of REM sleep; Obál et al., 1985. In the present study, this criterion was modified, REM sleep latency was defined as the time between sleep onset and the occurrence of the first at least 40-s long episode of REM sleep. Sleep onset was defined as the as the onset of the 1st at least 120-s long sleep epoch, as done by Cui et al., 2007) These values among the various (baseline, OUA post-OUA) recording days were compared by means of one-way ANOVA for repeated measures. The Bonferroni test was used for post hoc comparisons. Values of $\mathrm{p}<0.05$ were considered to be significant.

\section{Results}

The $2.5 \mu \mathrm{L} 2 \mathrm{mM}$ dose of OUA had a robust awakening effect, besides, epileptiform waves appeared in the EEG following administration of the drug. In order to avoid the induction of epileptiform waves the dose had to be substantially reduced. In a dose of $2.5 \mu \mathrm{L} 20 \mu \mathrm{M}$, OUA had only a moderate awakening action without inducing any epileptiform waves.

\subsection{Effects of $2.5 \mu L 2 \mathrm{mM}$ OUA}

Epileptiform waves (spikes and slow waves) appeared in the EEG in 10-15 min after the administration of $2.5 \mu \mathrm{L} 2 \mathrm{mM}$ OUA, and they often reappeared during the 12-h recording on the day of OUA administration $(41 \pm 11.6 \%$ of the 8-s intervals contained more or less epileptiform waves, usually only in a just noticeable amount, but sometimes these waveforms dominated the EEG; Fig. 1). No behavioral sign of epilepsy was noted, but it has to be mentioned that the behavior of the animals was not observed systematically. (In the lack of cameras in the recording chambers, such an observation was not possible without disturbing the sleep-wake activity of the rats.)

A robust arousing action was noted after administration of $2.5 \mu \mathrm{L}$ $2 \mathrm{mM}$ OUA (Fig. 2). The percentage of time spent in wakefulness displayed a diurnal (time of day) variation (ANOVA, $\mathrm{F}(3,15)=5.492$, $\mathrm{p}=0.0095)$. The amount (ANOVA, $\mathrm{F}(4,20)=8.929, \mathrm{p}=0.0003)$ and the daily distribution (ANOVA, recording day $\mathrm{x}$ time of day interaction, $\mathrm{F}(12,60)=6.593, \mathrm{p}<0.0001)$ of wakefulness varied among the different (baseline, OUA, post-OUA 1, 4 and 8) recording days. More time was spent in wakefulness during the day of OUA administration then on the baseline day (Bonferroni test, $p=0.0055$ ), wakefulness was enhanced in the initial post-administration 3-h period of the OUA day (Bonferroni test, $\mathrm{p}=0.0064$ ). The percentage of time spent in nonREM sleep had a diurnal (time of day) variation (ANOVA, $F$ $(3,15)=9.238, p=0.0011)$. The amount (ANOVA, $F(4,20)=6.766$, $\mathrm{p}=0.0013$ ) and the daily distribution (ANOVA, recording day $\mathrm{x}$ time

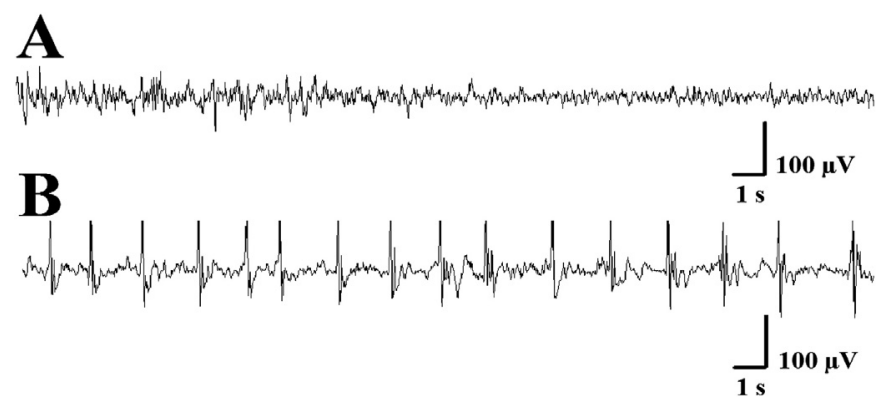

Fig. 1. Epileptiform EEG waves induced by $2.5 \mu \mathrm{L} 2 \mathrm{mM}$ OUA microinjection. A: non-REM sleep to wakefulness transition on the baseline day (control EEG recording); B: Epileptiform waves in the EEG on the OUA day. of day interaction, $\mathrm{F}(12,60)=7.934, \mathrm{p}<0.0001)$ of non-REM sleep varied among the different (baseline, OUA, post-OUA 1, 4 and 8) recording days. Non-REM sleep was suppressed in the initial post-administration 3-h period of the OUA day (Bonferroni test, $\mathrm{p}=0.0059$ ). The amount of REM sleep displayed a diurnal (time of day) variation (ANOVA, $\mathrm{F}(3,15)=9.369, \mathrm{p}=0.0009$ ). The percentage of time spent in REM sleep (ANOVA, $\mathrm{F}(4,20)=8.339, \mathrm{p}=0.0004$ ) and the daily distribution of REM sleep (ANOVA, recording day $\mathrm{x}$ time of day interaction, $\mathrm{F}(12,60)=2.342, \mathrm{p}=0.0154)$ varied among the different recording days. Less time was spent in REM sleep during the OUA (Bonferroni test, $\mathrm{p}=0.0154$ ) and the 1st post-OUA (Bonferroni test, $\mathrm{p}=0.0203$ ) days then on the baseline day, REM sleep was suppressed in the 2nd (Bonferroni test, $\mathrm{p}=0.0339$ ) and 3rd (Bonferroni test, $\mathrm{p}=0.0382$ ) post-administration 3 -h period of the day of OUA administration.

The number of wake (ANOVA, $\mathrm{F}(4,20)=13.027, \mathrm{p}<0.0001$ ), non-REM sleep (ANOVA, $\mathrm{F}(4,20)=11.990, \mathrm{p}<0.0001$ ), and REM sleep (ANOVA, $F(4,20)=6.567, p=0.0015$ ) epochs varied among the different (baseline, OUA, post-OUA 1, 4 and 8) recording days. The number of wake (Bonferroni test, $\mathrm{p}=0.0055$ ), non-REM sleep (Bonferroni test, $\mathrm{p}=0.0085$ ) and REM sleep (Bonferroni test, $\mathrm{p}=0.0447$ ) epochs were decreased on the day of OUA administration. (On the OUA day sleep-wake activity was dominated by long $\mathrm{W}$ epochs.) REM sleep latency also varied among the different recording days (ANOVA, $\mathrm{F}(4,20)=13.628, \mathrm{p}<0.0009$ ). REM sleep latency was elongated on the day of OUA administration (Bonferroni test, $\mathrm{p}=0.0010$ ), Table 1).

\subsection{Effects of $2.5 \mu L 20 \mu M$ OUA}

No epileptiform wave was induced by $2.5 \mu \mathrm{L} 20 \mu \mathrm{M}$ OUA.

This, low dose of OUA had a weak arousing action on the day of administration (Fig. 3). The amount of wakefulness displayed a diurnal (time of day) variation (ANOVA, $\mathrm{F}(7,49)=48.443, \mathrm{p}<0.0001$ ). The daily distribution of wakefulness varied among the different (baseline, OUA, post-OUA 1, 4 and 8) recording days (ANOVA, recording day $\mathrm{x}$ time of day interaction, $\mathrm{F}(28,196)=2.274$, $\mathrm{p}<0.0006$ ). Wakefulness was enhanced during the 12-h light period of the OUA day (Bonferroni test, $\mathrm{p}=0.0384$ ). No significant change was noted for any of the individual 3-h periods (Bonferroni test). This arousal effect was followed by an opposite reaction, wakefulness tended to decrease during the dark period. The amount of non-REM sleep had a diurnal (time of day) variation (ANOVA, $F(7,49)=70.556, \mathrm{p}<0.0001$ ). The daily distribution of non-REM sleep also varied among the different recording days (ANOVA, recording day $\mathrm{x}$ time of day interaction, $\mathrm{F}$ $(28,196)=1.866, \mathrm{p}<0.0077)$. The 12 -h or 3 -h values did not differ significantly from those of the corresponding baseline period (Bonferroni test). On the day of OUA administration, tendencies were noted in non-REM sleep to be decreased and increased during the light and dark 12-h periods, respectively. The amount of REM sleep displayed a diurnal (time of day) variation (ANOVA, $\mathrm{F}(7,49)=7.521$, $\mathrm{p}<0.0001$ ). The daily distribution of REM sleep varied among the different recording days (ANOVA, recording day $\mathrm{x}$ time of day interaction, $\mathrm{F}(28,196)=2.413, \mathrm{p}<0.0002)$. REM sleep was suppressed in the 12-h light period of the OUA day (Bonferroni test, $\mathrm{p}=0.0216$ ). No significant change was noted for any of the individual 3-h periods (Bonferroni test). Following the suppression in the light period, a tendency to a compensatory increase was noted during the dark period.

This low dose of OUA did not elicit any significant change in the other sleep parameters: SWA during NREMS; the number of wake, nonREM and REM sleep epochs; REM sleep latency (ANOVA), only tendencies to increase the numbers of wake and non-NREM sleep and to decrease the number of REM sleep epoch were noted on the OUA day (Table 2). 


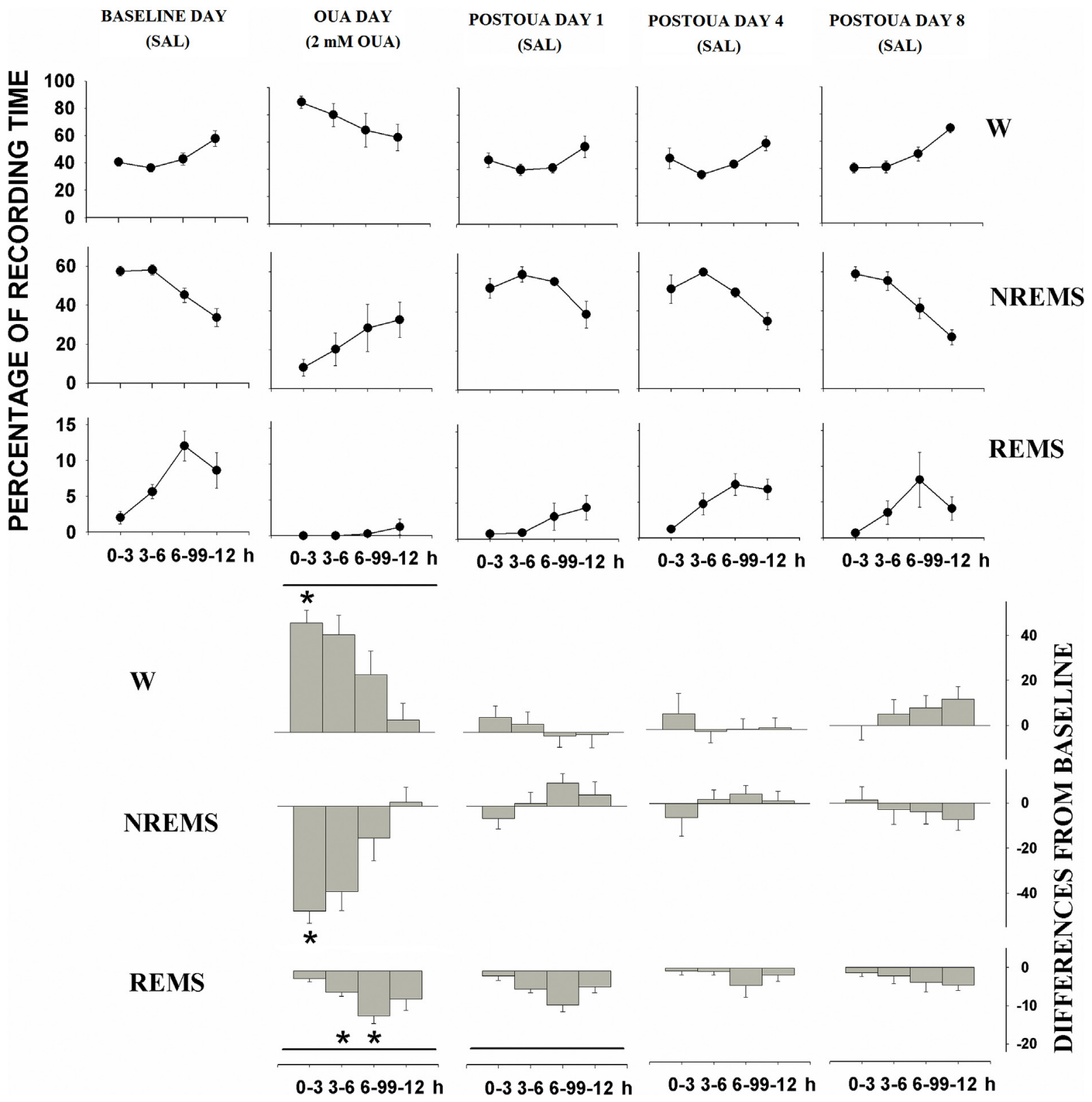

Fig. 2. Effects of $2.5 \mu \mathrm{L} 2 \mathrm{mM}$ OUA microinjection on sleep. The ICV microinjections were administered 5-15 min prior the onset of recordings. The top curves display the patterns of wakefulness (W), non-REM sleep (NREMS), and REM sleep (REMS) as the percentage of recording time on the baseline day (SAL), OUA day (OUA) and post-OUA days 1, 4, and 8 (SAL). The histograms represent the differences from baseline day values. Data are computed for consecutive 3-h bins (mean values \pm SE). The horizontal lines above (W) or below (REMS) the histograms denote significant differences between the given day and the baseline day (Bonferroni test, $\mathrm{p}<0.05$ ), the asterisk denotes significant differences of the 3-h values from baseline (Bonferroni test, $\mathrm{p}<0.05$ ).

Table 1

Effects of Effects of $2.5 \mu \mathrm{l} 2 \mathrm{mM}$ OUA microinjection: the numbers of W, NREMS and REMS epochs and the REM sleep latencies on the baseline day (SAL), OUA day (OUA) and postOUA days 1,4 and 8 (SAL), mean values \pm SE.

\begin{tabular}{lllll}
\hline & The number of W epochs & The number of NREMS epochs & The number of REMS epochs & REM sleep latency (min) \\
\hline Baseline day & $190.2 \pm 11.2$ & $194.0 \pm 12.0$ & $33.0 \pm 7.3$ & $91.7 \pm 27,9$ \\
OUA day & $74.0 \pm 20.1^{*}$ & $78.5 \pm 22.0^{*}$ & $1.8 \pm 1.6^{*}$ & $660.3 \pm 47.5^{*}$ \\
PostOUA day 1 & $146.7 \pm 18.7$ & $166.0 \pm 14.0$ & $15.5 \pm 7.9$ & $358.1 \pm 103.5$ \\
PostOUA day 4 & $173.5 \pm 19.0$ & $174.8 \pm 18.6$ & $28.7 \pm 5.5$ & $154.8 \pm 71.8$ \\
PostOUA day 8 & $178.3 \pm 12.5$ & $178.8 \pm 12.5$ & $15.7 \pm 4.6$ & $159.6 \pm 36.0$ \\
\hline
\end{tabular}

*p $<0.5$ (Bonferroni test). 


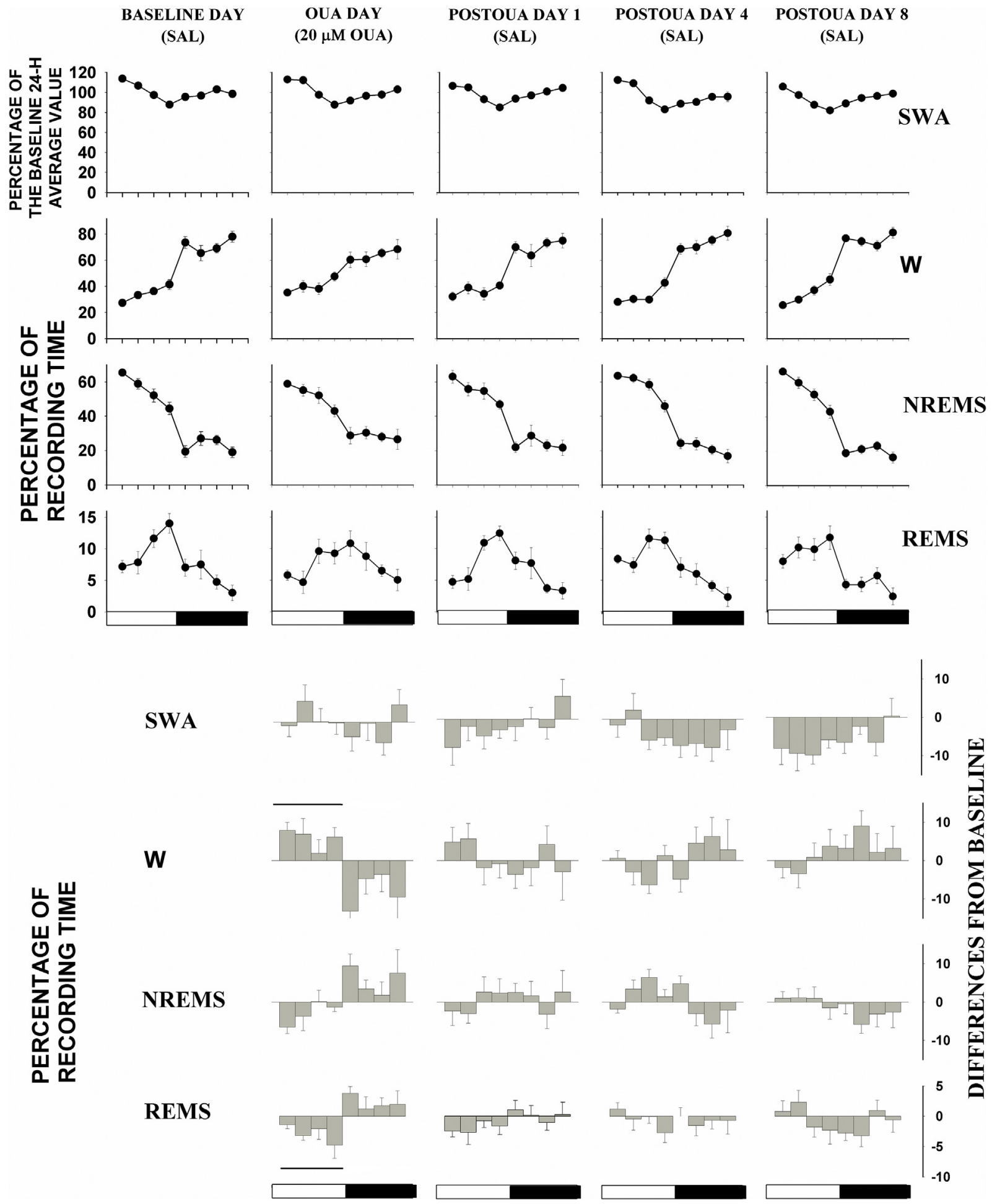

Fig. 3. Effects of $2.5 \mu \mathrm{L} 20 \mu \mathrm{M}$ OUA microinjection on the SWA and sleep. The ICV microinjections were administered 5-15 min prior to the onset of recordings. The top curves display the patterns of SWA during non-REM sleep (NREMS) as the percentage of baseline day 24-h mean value, wakefulness (W), non-REM sleep (NREMS), and REM sleep (REMS) as the percentage of recording time on the baseline day (SAL), OUA day (OUA), and post-OUA days 1, 4, and 8 (SAL). The histograms represent the differences from the baseline day values. The data are computed for consecutive 3-h bins (mean values \pm SE). The light and dark horizontal bars on the $\mathrm{X}$ axis indicate light and dark phases, respectively. The horizontal lines above (W) or below (REMS) the histograms denote significant differences between the light 12-h values of OUA day and the baseline (Bonferroni test, $\mathrm{p}<0.05$ ).

\section{Discussion}

The main finding of the present study was that suppression of NKA activity by both doses of OUA enhanced wakefulness. ICV administration of $5 \mu \mathrm{L} 1 \mathrm{mM}$ OUA exerts a long lasting influence on open field activity, which is still enhanced when the test is repeated several days after the administration of the drug (Ruktanonchai et al., 1998). In the present study, no similar long lasting effect on sleep was found. However, the open field test and sleep recording are performed in different conditions. In case of an open field test, the animal is placed into an 
Table 2

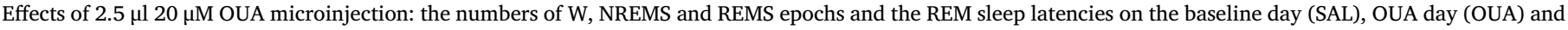
postOUA days 1,4 and 8 (SAL), mean values \pm SE.

\begin{tabular}{|c|c|c|c|c|}
\hline & The number of $\mathrm{W}$ epochs & The number of NREMS epochs & The number of REMS epochs & REM sleep latency (min) \\
\hline Baseline day & $294.3 \pm 15.4$ & $307.8 \pm 13.7$ & $80.3 \pm 7.2$ & $35.8 \pm 7.1$ \\
\hline OUA day & $342.0 \pm 16.3$ & $354.4 \pm 16.9$ & $73.8 \pm 9.5$ & $37.2 \pm 15.7$ \\
\hline PostOUA day 1 & $294.6 \pm 16.3$ & $304.6 \pm 15.6$ & $69.8 \pm 6.7$ & $49.4 \pm 8.4$ \\
\hline PostOUA day 4 & $288.8 \pm 13.8$ & $302.0 \pm 13.3$ & $77.6 \pm 8.0$ & $35.2 \pm 6.6$ \\
\hline PostOUA day 8 & $297.5 \pm 14.7$ & $310.8 \pm 12.7$ & $82.8 \pm 11.2$ & $46.9 \pm 10.1$ \\
\hline
\end{tabular}

open field equipment, i.e., into a new environment, whereas sleep recording is performed in the home cage. Our findings are in line with those of Kirshenbaum et al. (2011), who have reported enhancement of wake and suppressions of non-REM and REM sleep in genetically modified mice with decreased NKA activity. The enhancement of wakefulness in the present study may be related to an increase in neuronal excitability and activity, which is caused by a moderate decrease in NKA activity. The function of NKA is necessary for the excitability, but a moderate decrease in NKA activity can increase the excitability and the release of neurotransmitters (El Mallakh and Wyatt, 1995; McCarren and Alger, 1987). The appearance of epileptiform waves in the EEG following the administration of the high $(2.5 \mu \mathrm{L} 2 \mathrm{mM})$ dose of OUA is probably an indication of the increased excitability. Seizures have already been reported in response to OUA administration by Davidson et al. (1978). Interestingly, however, the suppression of endogenous OUA-like compounds by the administration of anti-OUA antibodies (such a treatment should decrease neuronal activity by increasing NKA activity) into the locus coeruleus, a nucleus containing noradrenergic REM-off neurons, decreases the amount of REM sleep (Jaiswal et al., 2010).

Additionally, sleep can influence NKA activity. REM sleep deprivation enhances neuronal NKA via noradrenaline and activation of alpha1-adrenergic receptors (Baskey et al., 2009). Phospholipase C and changes of intracellular calcium concentration are involved in the mediation of the noradrenaline induced enhancement of NKA (Amar and Mallick, 2018). By contributing to the maintenance of neuronal homeostasis, NKA might be involved in the homeostatic regulation of the sleep-wake activity. During a prolonged wake period, neuronal activity of the wake-promoting neurons rises. In case of neuronal activation, the intracellular accumulation of sodium, due to the influx of the ion during action potentials, results in an enhancement of NKA, which in turn decreases the excitability (El-Mallakh, 1983; Johar et al., 2014; Sims and Dale, 2014). The decrease in excitability may result in a drop of activity of the wake-promoting neurons leading to a sleep rebound following a long wake period. Wake-promoting, cholinergic neurons in the basal forebrain, which are also involved in the mediation of the arousing action of noradrenaline via alpha1-adrenergic receptors (Lelkes et al., 2013), have an important role in the induction of rebound sleep (Porkka-Heiskanen et al., 1997). Wake-associated increase in the activity of these neurons results in the release of adenosine, which inhibits the activity of these cholinergic cells and is involved in the induction of subsequent rebound sleep and the enhancement of SWA (Porkka-Heiskanen et al., 1997). Activity-dependent adenosine release may be linked to the activation of NKA (Sims and Dale, 2014). In case of neuronal activation, the enhanced NKA consumes ATP leading to a release of adenosine. Thus, blocking the NKA suppresses sleep, and sleep deprivation results in an enhancement of NKA, which may be involved in the induction of the subsequent sleep rebound. All these lines of evidence support the opinion that NKA may be involved in the regulation of the sleep-wake activity.

In addition to an enhancement of wakefulness, epileptiform waves appeared in the EEG in response to the high $(2.5 \mu \mathrm{L} 2 \mathrm{mM})$ dose of OUA. Though major depression is often accompanied by epilepsy, BD is rarely seen in epileptic patients (Schmitz, 2005). The lower $(2.5 \mu \mathrm{L} 20 \mu \mathrm{M})$ dose, without any epileptogenic action, appears to be a better model of
BD than the high one. Since the administration of OUA has been proposed as an animal model of $\mathrm{BD}$, changes of sleep-wake activity induced by this treatment should mimic those in mood disorders. In fact, wakefulness was enhanced by both doses of OUA in the present study. After an enhancement of wakefulness during the light 12-h period by the low dose (OUA was administered at light onset) tendencies to increase in sleep were noted in the following dark period, i.e., the loss of sleep in the light (rest) phase of the day was compensated during the dark (active) phase. Naps and circadian disturbances have been observed in BD (Riemann et al., 2002; Soreca, 2014). While the time spent in non-REM sleep tended to be reduced, a tendency to an increase in the number of non-REM sleep epochs was noted in response to the low dose of OUA, which might be a sign of fragmented NREM sleep. Fragmented sleep has been reported in mood disorders (Reynolds and Kupfer, 1987). Nonetheless, REM sleep latency was not found to be shortened in the present study. Shortening of REM sleep latency is also a characteristic feature of sleep disturbances in BD (Riemann et al., 2002). However, it is noteworthy that measuring REM sleep latency in animals is difficult, and it is not fully proved that the REM sleep latency measured in the present study is a real equivalent of the human REM sleep latency. In contrast to the present findings, a shortened REM sleep latency has been reported in genetically modified mice with decreased NKA activity (Kirshenbaum et al., 2011). In BD, the first non-NREM sleep period is shortened leading to a disinhibition and advanced occurrence of REM sleep (Riemann et al., 2002). No similar disinhibition and advanced occurrence of REM sleep or changes in sleep architecture was noted in response to OUA in the present study. Though sleep was suppressed by OUA, changes of sleep in BD were not really mimicked following the treatment with the drug. Whereas sleep in genetically modified mice is more similar to that in BD.

The present study was performed on rats. Though rats are nocturnal animals, otherwise their sleep is similar to that of diurnal ones. Despite of the diversity of the sleep characteristics of mammals there is no indication of fundamental differences in sleep among the various, diurnal and nocturnal, species (Tobler, 1995). Nocturnal animals are often used in sleep research, pharmacological sleep studies are often performed on rats. If it is taken to consideration that in rats the night and day are the active and rest phases, respectively, conclusions can be drawn even regarding the human sleep, though only with caution. Besides, homeostatic regulation of sleep is relatively independent of the circadian rhythm (Tobler, 1995). NKA might be involved in the homeostatic regulation of sleep. The effects of OUA on sleep architecture were also examined in the present study. There are notable similarities between the sleep architecture of rats and humans (Roncagliolo and Vivaldi, 1991).

The present findings give only limited support for the NKA hypothesis of BD. Though NKA has been found to be suppressed in BD in most of the studies, at least in one paper, increases have also been reported (Sengupta et al., 1980). There may be a link between NKA and the underlying mechanisms of $\mathrm{BD}$; however, it is possible that not necessarily the decrease in NKA activity itself is the important factor but the dysfunction of the regulatory mechanisms influencing NKA. These regulatory functions may be impaired in $\mathrm{BD}$ resulting in a decrease of NKA activity or a lack of compensatory increase. In fact, the lack of similar adaptive responses has been reported in BD. When lymphocytes 
of healthy subjects are incubated with lithium or ethacrynate, there is an increase in the number of NKA molecules. This response is missing in BD (Wood et al., 1991). Furthermore, the level of SP4 protein, a key enzyme in the activity dependent enhancement of NKA, is reduced in BD (Johar et al., 2014; Pinacho et al., 2011). These negative feedback processes influencing NKA are important for the maintenance of the homeostasis of neurons, and they are also involved in the homeostatic sleep regulatory mechanisms. The decrease in slow wave sleep in BD (Riemann et al., 2002) may mirror the dysfunction of these processes. This dysfunction may be a link between the underlying mechanisms of mood disorders and sleep disturbances. Since malfunctioning of the negative feedback regulation can also result in an oscillation of the regulated parameter, it may also be an explanation for the oscillation in mood in BD.

\section{Funding}

This research did not receive any specific grant from funding agencies in the public, commercial, or not-for-profit sectors.

\section{CRediT authorship contribution statement}

Zoltán Lelkes: Conceptualization, Data curation, Formal analysis, Investigation, Methodology, Project administration, Writing - original draft, Writing - review \& editing.

\section{Declaration of competing interest}

None.

\section{References}

Amar, M., Mallick, B.N., 2018. Mechanism of noradrenaline-induced +-1-adrenoceptor mediated regulation of Na-K ATPase subunit expression in Neuro-2a cells. Brain Res. Bull. 139, 157-166.

Baskey, G., Singh, A., Sharma, R., Mallick, B.N., 2009. REM sleep deprivation-induced noradrenaline stimulates neuronal and inhibits glial Na-K ATPase in rat brain: in vivo and in vitro studies. Neurochem. Int. 54, 65-71.

Cui, R., Li, B., Suemaru, K., Araki, H., 2007. Differential effects of psychological and physical stress on the sleep pattern in rats. Acta Med. Okayama 61 (6), 319-327.

Davidson, D.L.W., Tsukada, Y., Barbeau, A., 1978. Ouabain induced seizures: site of production and response to anticonvulsants. Can. J. Neurol. Sci. 5, 405-411.

El-Mallakh, R.S., 1983. The Na,K-ATPase hypothesis for manic-depression. I. General considerations. Med. Hypotheses 12, 253-268.

El-Mallakh, R.S., Adnan El-Masri, M., Huff, M.O., Li, X.P., Decker, S., Levy, R.S., 2003. Intracerebroventricular administration of ouabain as a model of mania in rats. Bipolar Disord. 5, 362-365.

El Mallakh, R.S., Wyatt, R.J., 1995. The Na,K-ATPase hypothesis for bipolar illness. Biol. Psychiatr. 37, 235-244.

Gamaro, G.D., Streck, E.L., Matte, C., Prediger, M.E., Wyse, A.T., Dalmaz, C., 2003. Reduction of hippocampal $\mathrm{Na}+, \mathrm{K}+$-ATPase activity in rats subjected to an experimental model of depression. Neurochem. Res. 28, 1339-1344.

Gulyani, S., Mallick, B.N., 1995. Possible mechanism of rapid eye movement sleep deprivation induced increase in Na-K ATPase activity. Neuroscience 64, 255-260.

Hodes, A., Rosen, H., Deutsch, J., Lifschytz, T., Einat, H., Lichtstein, D., 2016. Endogenous cardiac steroids in animal models of mania. Bipolar Disord. 18, 451-459.

Jaiswal, M.K., Dvela, M., Lichtstein, D., Mallick, B.N., 2010. Endogenous ouabain-like compounds in locus coeruleus modulate rapid eye movement sleep in rats. J. Sleep Res. 19, 183-191.
Johar, K., Priya, A., Wong-Riley, M.T., 2014. Regulation of Na + /K+-ATPase by neuronspecific transcription factor Sp4: implication in the tight coupling of energy production, neuronal activity and energy consumption in neurons. Eur. J. Neurosci. 39, $566-578$.

Keller, S., Frishman, W.H., 2003. Neuropsychiatric effects of cardiovascular drug therapy. Cardiol. Rev. 11, 73-93.

Kirshenbaum, G.S., Clapcote, S.J., Duffy, S., Burgess, C.R., Petersen, J., Jarowek, K.J., Yücel, Y.H., Cortez, M.A., Snead, O.C., Vilsen, B., Peever, J.H., Ralph, M.R., Roder, J.C., 2011. Mania-like behavior induced by genetic dysfunction of the neuron-specific $\mathrm{Na}(+), \mathrm{K}(+)$-ATPase +-3 sodium pump. Proc. Natl. Acad. Sci. U. S. A. 108, 18144-18149.

Lelkes, Z., Obál, F., Benedek, G., Rubicsek, G., Alföldi, P., Obál, F., 1987. Effects of acute and chronic treatment with an atypical antidepressant drug, nomifensine, on the sleep-wake activity in rats. Naunyn-Schmiedeberg's Arch. Pharmacol. 335, 149-153.

Lelkes, Z., Porkka-Heiskanen, T., Stenberg, D., 1994. Alpha1 adrenoceptor activation during REM sleep deprivation contributes to REM sleep rebound. J. Sleep Res. 3 (Suppl. 1), 145.

Lelkes, Z., Porkka-Heiskanen, T., Stenberg, D., 2013. Cholinergic basal forebrain structures are involved in the mediation of the arousal effect of noradrenaline. J. Sleep Res. 22, 721-726.

Li, R., El-Mallakh, R.S., Harrison, L., Changaris, D.G., Levy, R.S., 1997. Lithium prevents ouabain-induced behavioral changes: toward an animal model for manic depression. Mol. Chem. Neuropathol. 31, 65-72.

Looney, S.W., El-Mallakh, R.S., 1997. Meta-analysis of erythrocyte Na,K-ATPase activity in bipolar illness. Depress. Anxiety 5, 53-65.

Mallick, B.N., Singh, A., 2011. REM sleep loss increases brain excitability: role of noradrenalin and its mechanism of action. Sleep Med. Rev. 15, 165-178.

McCarren, M., Alger, B.E., 1987. Sodium-potassium pump inhibitors increase neuronal excitability in the rat hippocampal slice: role of a Ca2 +-dependent conductance. J. Neurophysiol. 57, 496-509.

Obál Jr., F., Benedek, G., Lelkes, Z., Obál, F., 1985. Effects of acute and chronic treatment with amitryptyline on the sleep-wake activity of rats. Neuropharmacology 24 (3), 223-229.

Obal Jr., F., Kapas, L., Gardi, J., Taishi, P., Bodosi, B., Krueger, J.M., 1999. Insulin-like growth factor-1 (IGF-1)-induced inhibition of growth hormone secretion is associated with sleep suppression. Brain Res. 818, 267-274.

Paxinos, G., Watson, C., 1998. The Rat Brain in Stereotaxic Coordinates. Academic Press, San Diego.

Pinacho, R., Villalmanzo, N., Lalonde, J., Haro, J.M., Meana, J.J., Gill, G., Ramos, B., 2011. The transcription factor SP4 is reduced in postmortem cerebellum of bipolar disorder subjects: control by depolarization and lithium. Bipolar Disord. 13, 474-485.

Porkka-Heiskanen, T., Strecker, R.E., Thakkar, M., Bjorkum, A.A., Greene, R.W., McCarley, R.W., 1997. Adenosine: a mediator of the sleep-inducing effects of prolonged wakefulness. Science 276, 1265-1268.

Reynolds, C.F., Kupfer, D.J., 1987. Sleep research in affective illness: state of the art circa 1987. Sleep 10, 199-215.

Riemann, D., Voderholzer, U., Berger, M., 2002. Sleep and sleep-wake manipulations in bipolar depression. Neuropsychobiology 45 (Suppl. 1), 7-12.

Roncagliolo, M., Vivaldi, E.A., 1991. Time course of rat sleep variables assessed by a microcomputer-generated data base. Brain Res. Bull. 27 (5), 573-580.

Ruktanonchai, D.J., El Mallakh, R.S., Li, R., Levy, R.S., 1998. Persistent hyperactivity following a single intracerebroventricular dose of ouabain. Physiol. Behav. 63, 403-406.

Schmitz, B., 2005. Depression and mania in patients with epilepsy. 2005. Epilepsia 46 , $45-49$.

Sengupta, N., Datta, S.C., Sengupta, D., Bal, S., 1980. Platelet and erythrocyte-membrane adenosine triphosphatase activity in depressive and manic-depressive illness. Psychiatr. Res. 3, 337-344.

Sims, R.E., Dale, N., 2014. Activity-dependent adenosine release may Be linked to activation of $\mathrm{Na}(+)-\mathrm{K}(+)$ ATPase: an in vitro rat study. PloS One 9, e87481.

Soreca, I., 2014. Circadian rhythms and sleep in bipolar disorder: implications for pathophysiology and treatment. Curr. Opin. Psychiatr. 27, 467-471.

Tobler, I., 1995. Is sleep fundamentally different between mammalian species? Behav. Brain Res. 69 (1-2), 35-41.

Wirz-Justice, A., Van den Hoofdakker, R.H., 1999. Sleep deprivation in depression: what do we know, where do we go? Biol. Psychiatr. 46, 445-453.

Wood, A.J., Smith, C.E., Clarke, E.E., Cowen, P.J., Aronson, J.K., Grahame-Smith, D.G., 1991. Altered in vitro adaptive responses of lymphocyte $\mathrm{Na}+, \mathrm{K}+$-ATPase in patients with manic depressive psychosis. J. Affect. Disord. 21, 199-206. 